\title{
AUTORREGULACIÓN Y CARGA DE TRABAJO EN TELETRABAJO DURANTE LA PANDEMIA COVID-19
}

\author{
SELF-REGULATION AND WORKLOAD IN TELEWORKING DURING THE COVID-19 \\ PANDEMIC
}

\section{Carlos Ignacio Díaz-Cánepa*}

\begin{abstract}
Resumen: Con base a la revisión de la literatura científica disponible, se presenta una perspectiva de análisis del teletrabajo en el contexto de pandemia COVID-19. Se discute desde la ergonomía cognitiva, de la Teoría Histórico-Cultural de la Actividad, y de la teoría de procesos auto y co-regulatorios, la necesidad de integrar los factores estructurales, de las exigencias laborales, los recursos disponibles, así como de los potenciales conflictos entre el trabajo y el hogar, para comprender las estrategias de enfrentamiento empleadas y los efectos del teletrabajo. Se plantea la necesidad de soluciones que se hagan cargo de la diversidad de situaciones presentes.
\end{abstract}

Palabras clave: Teletrabajo, COVID-19, procesos regulatorios, carga de trabajo.

\begin{abstract}
Based on the review of the scientific literature available, a perspective of analysis of teleworking in the context of the COVID-19 pandemic is presented. The need to integrate structural factors, work demands, available resources, as well as potential work-home conflicts, for the understanding of coping strategies, and telework effects, is discussed from cognitive ergonomics, Cultural-Historical Activity Theory, and the self and co-regulatory processes theory. The need for solutions that take care of situations diversity arises.
\end{abstract}

Keywords: Telework, COVID-19, regulatory process, workload.

Recepción: 14.10.2021 / Revisión: 25.10.2021 / Aceptación: 30.11.2021

\section{Introducción}

El teletrabajo estaría esencialmente definido por la distancia espacial entre trabajadores y la actividad mediada por TIC, incorporando grados variados de interdependencia entre tareas, trabajos de distinta naturaleza, diversos entornos tecnológicos y distancias temporales, en donde la virtualidad y la distribución del trabajo entre distintos actores, podrían presentarse en distintos continuos (Henry et al., 2021). El paso desde el trabajo presencial al teletrabajo, ha implicado para los trabajadores, transitar desde una situación de distribución del trabajo basado en la diferenciación de roles, y secuencias temporales, a una en que se ha introducido además de una amplia distribución espacial de los trabajadores, una extensa mediación instrumental del trabajo basado en TIC (Ruiller et al., 2017). También ha significado un giro

*Departamento de Psicología, FACSO, Universidad de Chile. Santiago, Chile. Correo electrónico: carldiaz@uchile.cl. Orcid: https://orcid.org/oooo-0003-1017-287X 
paulatino del foco de la evaluación del trabajo realizado por los trabajadores, desde un énfasis en el control horario, hacia uno centrado en los resultados (Van der Lippe \& Lippévyi, 2019).

Existirían diferentes perspectivas sobre la relación entre el teletrabajo y las prácticas de trabajo. Por una parte, se asumiría que la naturaleza del trabajo no sería influenciada por las prácticas de trabajo remoto, por lo que el arreglo de su implementación podría definirse principalmente por criterios de gestión. Un segundo enfoque asumiría que, en el trabajo remoto, la mayoría de las tareas, comunicaciones y colaboraciones interpersonales estarían mediadas por las TIC, y que esto podría cambiar significativamente las demandas laborales, la autonomía y aspectos relacionales del trabajo. Una tercera perspectiva, propondría que el trabajo remoto operaría como un contexto, en lugar de actuar como una variable independiente, por lo que las características del trabajo a distancia deberían adaptarse a la nueva forma de trabajar para lograr un mejor desempeño y bienestar, lo que podría constituirse en una oportunidad para modificar las estructuras tradicionales del trabajo, asociadas al trabajo presencial (Wang et al., 2020), incentivando a que el teletrabajo tienda a construirse con base a un orden social negociado, considerando las nuevas disposiciones del espacio y el tiempo (Sewell \& Taskin, 2015). Es esta tercera perspectiva la que anima este texto.

La pandemia por COVID-19 ha implicado una importante expansión del trabajo remoto, trasladando en forma masiva la actividad laboral desde la presencialidad al teletrabajo (Chong et al., 2020). Así, el actual escenario ha significado: a) una extensión de esta práctica a nuevos grupos; b) una expansión creciente del teletrabajo hacia el tiempo libre, fuera del horario normal de trabajo, compitiendo con la vida privada, dada su rápida e improvisada implementación; y c) una expansión de las tecnologías de la información y la comunicación (TIC) en uso, diluyendo las fronteras tempo-espaciales (Thulin et al., 2019; Hughes \& Silver, 2020).

Las características personales más importantes que se señalan para el teletrabajo, incluyen autodisciplina, la capacidad de trabajar solo, conocimientos tecnológicos, habilidades de comunicación, la autoeficacia, la autonomía, el control del trabajo y la autogestión (Wang et al., 2020; Fischer et al 2021; Errichiello \& Paniese, 2021). Sin embargo, la incorporación forzada y masiva de nuevos grupos de trabajadores al teletrabajo, ha desbordado el supuesto de gestión de recursos humanos, en que el trabajo remoto estaría reservado para personas que cuenten con flexibilidad cognitiva, conductual y adaptabilidad (Ruiller et al, 2017), haciendo crucial la alineación de las demandas y recursos organizacionales con las circunstancias en las que se desenvuelven las vidas individuales (Donnelly \& Proctor-Thomson, 2015).

Previo a la pandemia por COVID-19, se ha documentado en Chile que el paso desde la presencialidad a un formato online, ha traído valorados beneficios para los trabajadores, tales como la reducción de los desplazamientos entre el hogar y el lugar de trabajo, pero en paralelo se han reconocido efectos negativos, asociados al incremento combinado de las demandas laborales y del hogar, y una emergente merma en los procesos de socialización y de integración organizacional. Los teletrabajadores que se encontraban en trabajo remoto previo a la pandemia por COVID-19, lo hacían mayoritariamente en formato de home office, 
cumpliendo de 1 a 3 días de teletrabajo (Olivares et al., 2020). Por lo general, este perfil de trabajador remoto se encontraría mayoritariamente en áreas de gestión, de análisis, o soporte, y asumieron este formato de trabajo a través de procesos concordados con sus empleadores. En contraste, hoy los teletrabajadores "nuevos" realizan actividades más heterogéneas, asociadas con tareas de naturaleza más administrativas, de servicios, comerciales y rutinarias (Thulin et al., 2019), y mayormente forzados por las circunstancias. Tal distinción resulta relevante desde el punto de vista del desarrollo de procesos autorregulatorios, pues la experiencia previa de teletrabajo, sería un recurso desigualmente disponible, y el control sobre los objetivos y modos de realización de las tareas, se encontraría heterogéneamente presente entre estos grupos. Del mismo modo, la naturaleza abrupta y no voluntaria en la que se ha adoptado el teletrabajo por parte de muchas personas, constituye un contexto ineludible para comprender el modo como estas han enfrentado las nuevas exigencias que esta modalidad conlleva.

Se ha documentado que existirían igualmente diferencias entre trabajadores remotos a tiempo completo, y trabajadores remotos de baja intensidad. Los primeros darían más importancia a ser apoyados por otros trabajadores remotos con quienes tienen relaciones preexistentes, mientras que los segundos valorarían preferentemente el apoyo brindado por colegas que trabajan desde la oficina (Wang et al., 2020; Errichiello \& Pianese, 2021). Estos últimos, podrían verse además negativamente impactados por el aumento de la carga de trabajo, ya que las personas que no trabajan a distancia, deberían a menudo asumir responsabilidades que no pueden ser asumidas por los teletrabajadores (Gajendran et al., 2015).

También se ha señalado que durante la pandemia muchas empresas han subestimado la importancia, o no han tenido el tiempo suficiente, para definir prácticas y herramientas para apoyar a los teletrabajadores, tales como soporte tecnológico, información oportuna, materiales de trabajo relevantes, y autoridad para tomar decisiones. El agotamiento que produciría trabajar bajo el contexto de la pandemia por COVID-19, se expresaría a menudo en secuencias de días de trabajo agotador, alternados con días de abstinencia laboral. Esto habría incidido en las interpretaciones de los trabajadores respecto a cuánto la organización valora sus contribuciones y bienestar, situando esta percepción como trasfondo de este comportamiento organizacional. (Chong et al., 2020; Errichiello \& Pianese, 2021).

En este contexto, parece relevante no solo identificar y ponderar los impactos del teletrabajo sobre las personas que se han sumado recientemente a esta modalidad, sino que importa igualmente identificar cómo los teletrabajadores han ido enfrentando dichas demandas para dar respuesta a sus metas laborales, personales, del hogar, y cómo dichas modalidades se relacionarían con la carga de trabajo resentida.

\section{Materiales y métodos}

Se ha realizado una revisión de la literatura científica disponible sobre el teletrabajo, con base a la cual se han seleccionado 35 artículos, atendiendo a criterios de pertinencia, para dar cuenta de la diversidad de situaciones reportadas en este campo. El análisis de estas 
situaciones se ha efectuado a partir de los aportes de la ergonomía cognitiva, la Teoría Histórico-Cultural de la Actividad (THCA), y la teoría de procesos auto y co-regulatorios. La integración de los antecedentes analizados, se han traducido en modelos orientados a soportar el análisis de la actividad en teletrabajo, y el desarrollo de alternativas de intervención, diseño y arreglo de situaciones de teletrabajo.

\section{Resultados y discusión}

\section{Teletrabajo, regulación, autorregulación y co-regulación}

Esquemáticamente, el teletrabajo plantearía particulares tensiones en razón de tres fuentes principales de exigencias: a) la sobreposición de los espacios y de las temporalidades en que se desarrollan respectivamente la actividad laboral y la actividad del hogar; b) la redefinición de las formas que asume la actividad en este contexto en relación a aquellas desplegadas en formato presencial, afectando a menudo la jerarquía de relevancia de los contenidos de las tareas, las interacciones, el status de los roles, etc., y c) la redefinición de los roles, uso de espacios, gestión de las temporalidades, cuidado de las relaciones, etc., del hogar (figura 1).

Figura 1. Tensiones entre los sistemas de actividad laboral y hogar basado en el sistema de actividad de Engeström (2008).

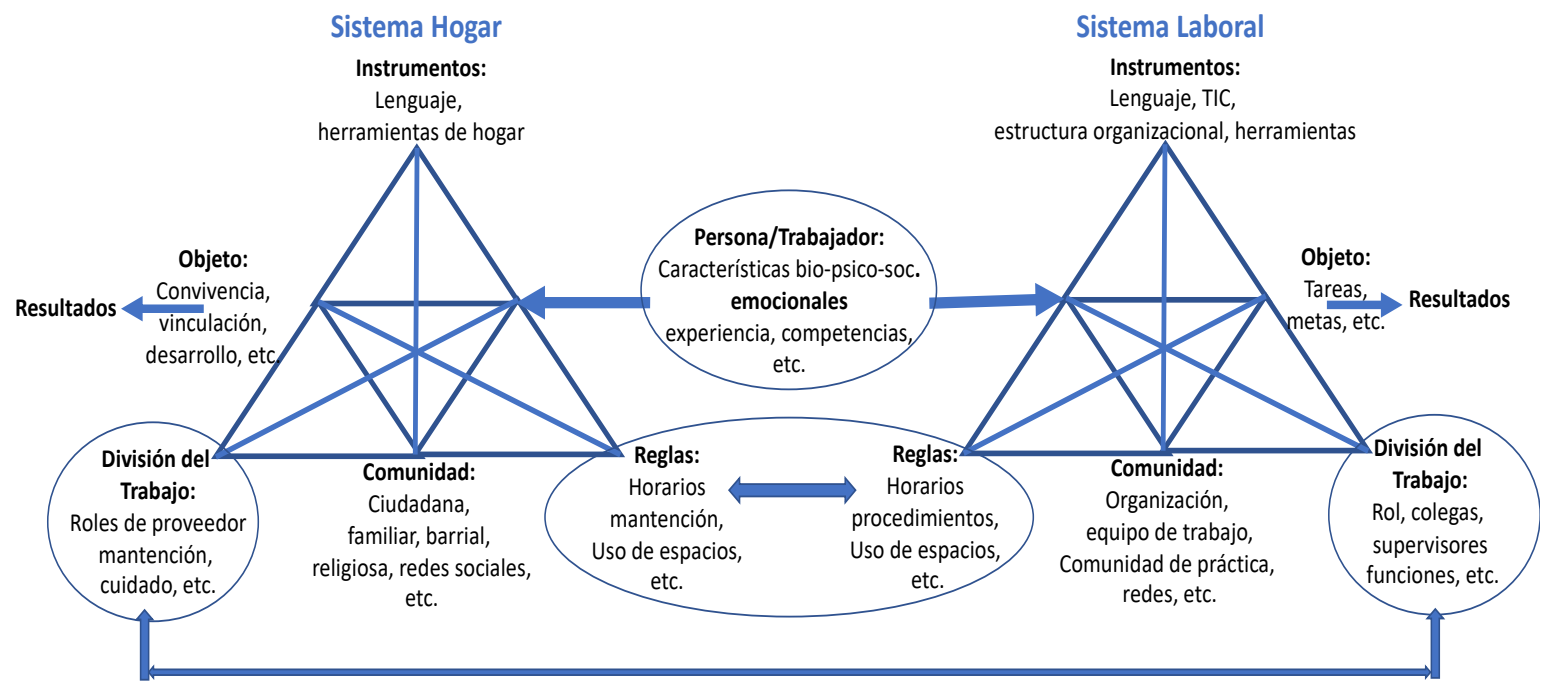

En las actuales condiciones, los teletrabajadores se han visto mayormente interpelados a recurrir a nuevas competencias, capacidades y recursos materiales e instrumentales, para desarrollar formas de modulación de las demandas del trabajo y del hogar. Estas, sin embargo, probablemente se encuentren desigualmente distribuidas y posibilitadas entre las personas y sus contextos de actividad, afectando por lo tanto de modo desigual la carga de trabajo y estrés resentidos por estas, así como la calidad y resultados de su trabajo.

Existirían variados factores incidentes que contribuirían a mitigar, y eventualmente a potenciar el desarrollo de la actividad laboral, tal como la capacidad de autorregulación y de co-regulación que las personas logren desplegar para enfrentar las distintas exigencias de la tarea y del hogar (Morosanova et al., 2017; Zheng et al., 2020). El cambio desde un escenario 
presencial al de actividad remota implicaría, por lo tanto, el despliegue de importantes esfuerzos adaptativos y ajustes en los procesos autorregulatorios.

La autorregulación no consiste en un mero proceso homeostático, sino que, en un proceso dirigido a un objetivo, que tiene funciones sistémicas integradoras (Sanda, 2018). La autorregulación puede ser entendida como una secuencia, en la que las personas definen la tarea, establecen metas, elaboran estrategias, planifican o prevén lo que requieren para la ejecución de la tarea, se motivan a sí mismos, y a continuación ponen en práctica sus planes y estrategias (Winters et al., 2008). Así, la cognición, el comportamiento y la motivación estarían integrados y organizados por mecanismos de autorregulación orientados al logro de metas conscientes (Bedny \& Karwowski, 2006). Esta actividad orientada a metas, determinaría la especificidad de la interacción de los sujetos con el mundo externo. Durante esta interacción, los procesos mentales evolucionarían expandiéndose (Engeström, 2008). No obstante, tal proceso no necesariamente tomaría siempre una forma consciente, pues con base a la experiencia, las estrategias empleadas en la autorregulación, tenderían a automatizarse, y a desplegarse de manera no consciente, induciendo una reducción de la carga cognitiva (Wirth, et al., 2020). Desde la perspectiva laboral, la autorregulación sería entonces, una modalidad intrínseca a la naturaleza teleológica de las acciones que los trabajadores deben desplegar y articular durante su actividad (Sanda, 2018). Esto, en la medida en que las personas autorregulan sus estrategias y el alcance de su relación con su entorno externo (Bedny \& Karwowski, 2006).

Estos procesos, operarían al interior de particulares marcos prescriptivos regulatorios, tales como la supervisión y reglas organizacionales que encuadran el comportamiento de los miembros de la organización (horarios, evaluación del desempeño, etc.). La regulación, sería una influencia externa en un sistema, mientras que la autorregulación sería una influencia desde dentro del sistema de actividad (Bedny et al., 2000). La co-regulación, por su parte, resultaría de las interacciones entre actores que participan o inciden sobre la actividad remota. En los colectivos de trabajo, la co-regulación incidiría sobre la cognición compartida, el modo de operar y la retroalimentación dentro del contexto social compartido, articulando el conocimiento metacognitivo, las habilidades y experiencia regulatoria distribuida al interior de los colectivos (Annosi, 2017). La co-regulación estimularía, ampliaría y eventualmente constreñiría la autorregulación, expandiendo las dimensiones cognitivas y sociales de las personas en los colectivos de trabajo (Volet et al., 2009; Hadwin et al., 2017). No obstante, los procesos co-regulatorios no responderían necesariamente a intenciones negociadas y construidas conscientemente en torno a metas comunes, sino que podrían derivar de modulaciones contextuales, sin mediar necesariamente concertación entre los actores. Así, la co-regulación no implicaría necesariamente cooperación, en el sentido de implicar al conjunto de los miembros de un colectivo de trabajo en torno a metas, y la naturaleza e intensidad de la co-actividad resultantes, pueden ser variables y heterogéneas (de la Garza \& Weill-Fassina, 2000). Esto implicaría resolver la tensión entre las regulaciones necesarias para articular los colectivos, y aquellas requeridas para articular las tareas, cuando estas dimensiones de la actividad, no responden necesariamente a las mismas condiciones, exigencias, orientaciones y dinámicas (Chan, 2012). Es en esto que las regulaciones organizacionales resultan claves para fijar condiciones de borde e indicaciones referenciales, tanto para la autorregulación, como para la co-regulación (figura 2). 
Figura 2. Regulación, autorregulación y co-regulación en el sistema de actividad basada en Engeström, (2008) y Zheng et al. (2020).

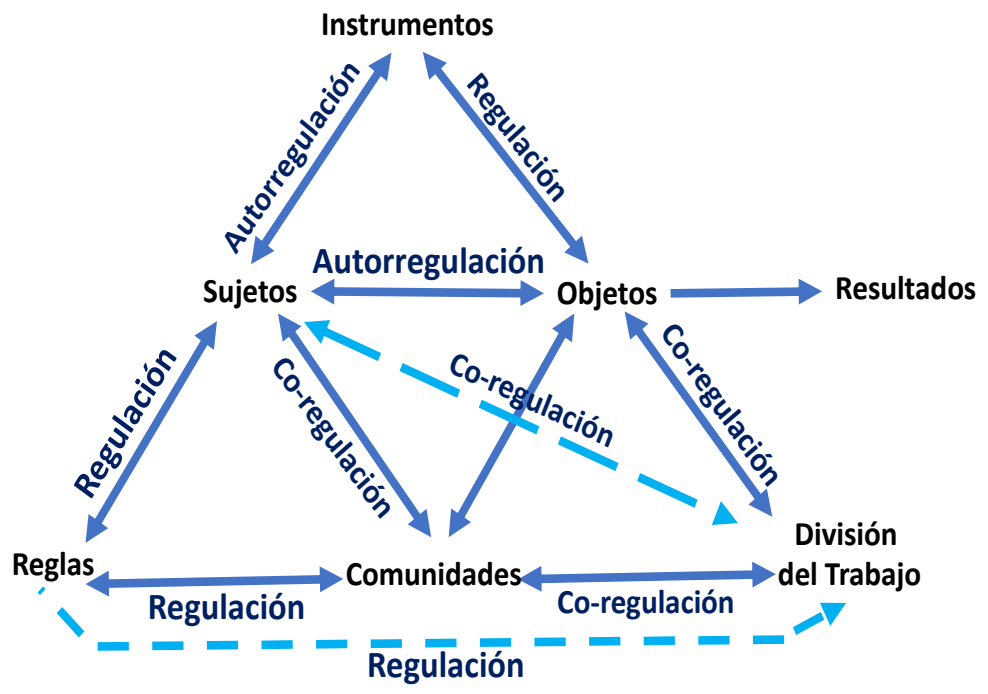

En lo específico, en el plano laboral y organizacional, el teletrabajo interpela la aparición de tensiones respecto a los roles y modos de resolver la relación entre supervisión y teletrabajadores, entre teletrabajadores, así como entre teletrabajadores y trabajadores presenciales, con los que deben eventualmente coordinar o articular actividades. En este contexto, aquellos que desarrollan tareas con mayor interdependencia con sus compañeros de trabajo, habitualmente requerirían compartir modelos mentales de cómo realizar las tareas de manera interdependiente, y experimentarían en consecuencia mayor agotamiento al final del día producto del esfuerzo asociado (Chong et al., 2020). Frente a las dificultades, todo el grupo de trabajadores se vería involucrado, requiriendo no solo la inversión de sus recursos personales, sino que también contar con una serie de recursos para comunicar, coordinar y sincronizar esfuerzos. Resolver colectivamente las situaciones mientras trabajan distribuidos en diferentes lugares, y eventualmente desfasados en el tiempo, se volvería problemático, dependiendo de reuniones programadas, y con bajas posibilidades de intercambio de conocimientos tácitos (Golden \& Raghuram, 2010). Así, los teletrabajadores se enfrentarían a un desafío cuando en el transcurso de las actividades compartidas, deben co-orientarse hacia perspectivas o enfoques adoptados por otros (Golden, 2007).

\section{Rol y uso de las temporalidades}

El manejo del tiempo, es sin duda uno de los elementos claves en los procesos de autorregulación y co-regulación, y en la actividad de teletrabajo ocupa una posición central. Las distintas regulaciones implican una adaptación cíclica, ya que responden a una serie de contingencias en el tiempo (Winne, 2019). El modo de enfrentar esas contingencias, incidiría en los efectos del trabajo sobre las personas, y en particular sobre la carga de trabajo resentida. Considerar las dimensiones temporales del trabajo remoto implica en buena medida, identificar los tipos, contenidos, orden, oportunidad y como los y las teletrabajadores y teletrabajadoras usan las estrategias de trabajo (Chan 2012). Esto, en el entendido que la autorregulación de la actividad siempre incorpora mecanismos de anticipación, sobre los que se definen las estrategias de ejecución de las tareas (Bedny \& 
Karwowski, 2006), y que el orden de los pasos a seguir, así como su pertinencia y oportunidad, son relevantes en los resultados de la actividad.

Se han reportado hallazgos contradictorios en la relación entre el teletrabajo y el control del tiempo. Por una parte, se señala la existencia de ventajas del teletrabajo en términos de tranquilidad y control del uso del tiempo a corto plazo, mientras que, en el teletrabajo colectivo y a largo plazo, se intensificarían las expectativas de disponibilidad, reduciendo la capacidad de los participantes para desconectarse, y generando de paso, sentimientos de un menor control del tiempo (Mazmanian et al., 2013). A su vez, se ha señalado que muchos teletrabajadores emplean el tiempo para crear patrones $o$ comportamientos monocrónicos, donde el tiempo se considera lineal, de modo que las tareas ocurran sin interrupción, mientras que otros usan el tiempo para organizar patrones o comportamientos policrónicos, superponiendo e intercalando interacciones y ocupaciones, en función de rutinas temporales relacionadas, por ejemplo, con los horarios escolares de sus hijos (Fonner\& Stache, 2012). El teletrabajo permitiría a algunas personas reducir la presión temporal, mientras que, para otras personas, el teletrabajo incrementaría el sentimiento de presión temporal (Peters y van der Lippe, 2007). Estas diferencias dependerían parcialmente de la naturaleza y de la práctica específica de teletrabajo (contenido de las tareas, extensión y distribución del tiempo, modalidades de trabajo, etc.), y si este responde a una crisis ad hoc, o una actividad a largo plazo (Peters et al., 2009). Así, no habría necesariamente relación entre la naturaleza de las funciones desempeñadas por los teletrabajadores con la presión temporal percibida, sino que esta tendría una conexión con el tiempo que le dedican. Aquellos que solo laboran durante las horas de trabajo regulares, experimentarían menos presión de tiempo en comparación con aquellos que lo hacen además fuera de las horas regulares de trabajo. Tener hijos pequeños en el hogar y ser mujer, aumentaría la presión de tiempo percibida, mientras que a medida que aumenta la edad, la percepción de presión temporal disminuiría. Este último factor tendría un efecto mayor sobre las posibilidades de control del tiempo, que los factores laborales, los que presentarían características de mayor estabilidad, en tanto que restricciones fijas, posibilitando su mayor control (Thulin et al., 2019).

El teletrabajo se ha asociado con un aumento de la productividad, pero podría producir el efecto contrario a largo plazo si el ritmo y la extensión temporal del trabajo no son los adecuados. Se ha señalado que la autonomía en la gestión del tiempo de trabajo, estimularía el trabajo nocturno y un incremento de las horas de trabajo (Dumas \& Ruiller, 2014). El teletrabajo, por el distanciamiento que genera entre los participantes, presentaría inconvenientes respecto a la formación de equipos cohesionados, lo que acarrearía efectos negativos para el desempeño, pues este estaría influenciado no solo por el trabajo de cada teletrabajador en particular, sino también por la interacción con sus compañeros que también trabajan desde sus casas. En consecuencia, el rendimiento de los equipos sería peor en la medida que más compañeros de trabajo se encuentren en trabajo remoto. Esto, porque el mayor volumen de las interacciones necesarias para articular los equipos de trabajo atentaría contra el rendimiento colectivo (Van der Lippe \& Lippéyi, 2019).

Instalándose el teletrabajo como una modalidad de ejercicio de la actividad laboral a largo plazo, parece importante entonces, prestar atención a los procesos de desarrollo y 
adquisición de estrategias y herramientas, que le permitan a los teletrabajadores ir autorregulando y co-regulando las exigencias y el despliegue de sus recursos y acciones en el tiempo, pues el sistema de regulación de las personas y los colectivos tomaría forma y se transformaría en largos períodos (Sanda, 2018).

\section{Regulaciones organizacionales, instrumentales y contextuales}

Taskin (2006), propone el concepto de "despacialización", para referirse a la distancia física y psicosociológica inducida por la práctica del teletrabajo, que alteraría el método de gestión. La distancia percibida incidiría sobre la construcción social y su dimensión simbólica, con base a la cual los equipos dispersos, implementarían nuevas prácticas, las que podrían ser incentivadas, o eventualmente limitadas por la organización del trabajo (Ruiller et al., 2017).

Por la pandemia COVID-19, las organizaciones se han visto en la necesidad de revisar contingentemente sus reglas y prácticas, para hacer frente a los frecuentes giros de los acontecimientos. Los teletrabajadores, pueden recibir nuevas tareas e instrucciones contradictorias en diferentes días, alterando la forma en la que las realizaban (Chong et al., 2020), por lo que, las reglas y la organización del trabajo tendrían un papel importante en el éxito del teletrabajo (Dumas y Ruiller, 2014). A su vez, los lugares de trabajo constituyen procesos de gestión en sí mismos, al facilitar la "visibilidad" y "presencia", promoviendo la posibilidad de que los supervisores y otras personas observen a los trabajadores, y a su vez, permitiendo participar a los trabajadores en relaciones con sus compañeros de trabajo (Felstead, 2003). Trabajar desde casa crearía problemas para ambos aspectos del control administrativo, e implicaría revisar los modos de realizar la gestión (Ruiller et al., 2017). Los supervisores de trabajo remoto tenderían a orientarse prioritariamente a encuadrar las tareas, más que a un estilo de liderazgo orientado a las relaciones. Según Madlock (2012), este estilo aparecía como un mayor predictor de la satisfacción comunicativa, de la satisfacción laboral y el compromiso organizacional, ya que los teletrabajadores se sentirían más cómodos con este tipo de liderazgo, pues ellos mismos estarían principalmente orientados a la tarea. Igualmente, la gestión de los estilos y contenidos comunicacionales sería un aspecto clave a definir desde la supervisión, ya que las herramientas de trabajo remoto, podrían también operar con base a una visión cultural, en tanto que soporte de la dinámica interpersonal, y no solo como un medio performativo. Esto, resultaría particularmente relevante en casos en que la supervisión se realice desde una posición de trabajo presencial pues, cuando el supervisor no comparte la experiencia de teletrabajo, podrían acentuarse los riesgos de sentimientos de aislamiento por parte de los teletrabajadores e incrementar las ocasiones de mala interpretación de los mensajes (Ruiller et al., 2017). En este sentido, es posible entender que la supervisión jugaría una influencia tanto en el plano regulatorio, como en el co-regulatorio, pues podría operar en el encuadramiento de las tareas, a la vez que participa de los procesos relacionales con los teletrabajadores.

Las dificultades para establecer cuáles serían las modalidades apropiadas de supervisión del trabajo remoto, han motivado el desarrollo de estrategias de sustitución o neutralización de estas funciones por otros medios. Por ejemplo, la orientación a la tarea o a las relaciones, podrían ser actuadas por los propios teletrabajadores, de manera a sustituir la influencia de los supervisores, o por medio de generar "vacíos de influencia", con base a 
regulaciones organizacionales, la claridad de las tareas, la experiencia y competencias de los teletrabajadores o la fortaleza de los equipos de trabajo, entre otros (Dahlstrom, 2013). Un aspecto a considerar desde esta óptica, es el de la forma en que se cristalizan y se muestran, las lógicas, procedimientos y soportes del trabajo en los instrumentos técnicos que disponen los teletrabajadores para desarrollar su actividad (Rabardel, 1995).

Se ha señalado que, para el trabajo en equipo, la presencia digital no puede compensar la presencia corporal (Van der Lippe \& Lippéyi, 2019), aunque esto dependería de la naturaleza del trabajo a realizar por los teletrabajadores, y las prácticas instaladas en los equipos de trabajo (Ruiller et al., 2017). Tales prácticas, constituirían un factor relevante para la identificación de los miembros con sus equipos de trabajo, ya que las TIC, como infraestructura de control, podrían transmitir una naturaleza opresiva, intrusiva y de vigilancia de la actividad de los teletrabajadores (Felstaed, 2003). Esto ha sido asociado como fuente de malestar, y de desarrollo de comportamientos de resistencia por parte de teletrabajadores (Nansen et al., 2010). No obstante, los mediadores técnicos empleados en el teletrabajo serían relevantes para el quehacer, pues el apoyo necesario para orientar y apoyar la autorregulación de la actividad, podría provenir del entorno tecnológico disponible, y no solamente de los compañeros (Järvelä, et al., 2016; Hadwin et al., 2017). Aquellos teletrabajadores que cuentan con un alto apoyo tecnológico participarían mayormente en intercambios de conocimientos y tendrían menos obstáculos para la colaboración, que aquellos que cuentan con poco apoyo tecnológico (Golden \& Raghuram, 2010). La tecnología podría jugar un rol de herramienta metacognitiva, facilitando los procesos co-regulados, mediante su potencial para promover el desarrollo de una cognición compartida o, al contrario, si su diseño y funcionalidad no soportan adecuadamente las mediaciones implicadas en la actividad colectiva, delimitándola y constriñéndola (Hadwin et al., 2017). El uso de las TIC en el teletrabajo no sería solo el de un medio de comunicación, sino que también sería un medio de reconfiguración del trabajo (Baker et al., 2006).

En el escenario del teletrabajo, los procesos autorregulatorios se encuentran mediados de modo importante por instrumentos auxiliares externos, promoviendo el desarrollo tanto, de esquemas que permitan identificar contextos y modalidades pertinentes de actividad, como de modulación del comportamiento (Vygotsky, 1981). Las TIC, al igual que los distintos soportes y dispositivos (agendas, ayuda memoria, calendarios...) no solo permitirían "trabajar en cualquier momento y en cualquier lugar" (Hughes \& Silver, 2020), sino que también incidirían en la forma y contenidos de la propia actividad, operando ya sea como herramientas principales, complementarias o sustitutas de las funciones a ejecutar (Díaz, 2008). En ese contexto, los instrumentos técnicos, en análogo título que los instrumentos psicológicos, asumirían una función de mediación entre el individuo y la realidad, así como del individuo consigo mismo (Vygotsky, 1997). El uso, y los contextos de uso de instrumentos simbólicos y materiales, serían entonces, aspectos claves para entender la forma en que se construyen y despliegan los procesos de autorregulación de la actividad (Nückles et al., 2020), y como estos inciden sobre la carga de trabajo resentida.

Así, el rol articulador de la supervisión y de los artefactos de soporte de la actividad, sería un factor clave (Zheng et al., 2020), por lo que la apropiación por parte de los supervisores y sus equipos de las herramientas de comunicación remota, y el desarrollo de 
procesos de co-construcción de nuevas rutinas organizacionales, serían centrales para orientar adecuadamente la actividad desplegada por los teletrabajadores (Ruiller et al., 2017). A su vez, cabe destacar en este escenario, que los méritos protectores del trabajo remoto serían en buena medida dependientes de un mayor control, autonomía y voz de los trabajadores.

\section{Teletrabajo, actividad instrumentada, procesos regulatorios, metas y carga de trabajo}

El análisis de la actividad instrumentada orientada a metas implica considerar los propósitos que motivan a los teletrabajadores, las estrategias que emplean para llevarlos a cabo, los recursos personales e instrumentales que ponen en juego, las condiciones técnicas, organizacionales, estructurales y contextuales, así como los efectos de su actividad (figura 3).

Figura 3. Esquema general del proceso de actividad en teletrabajo.

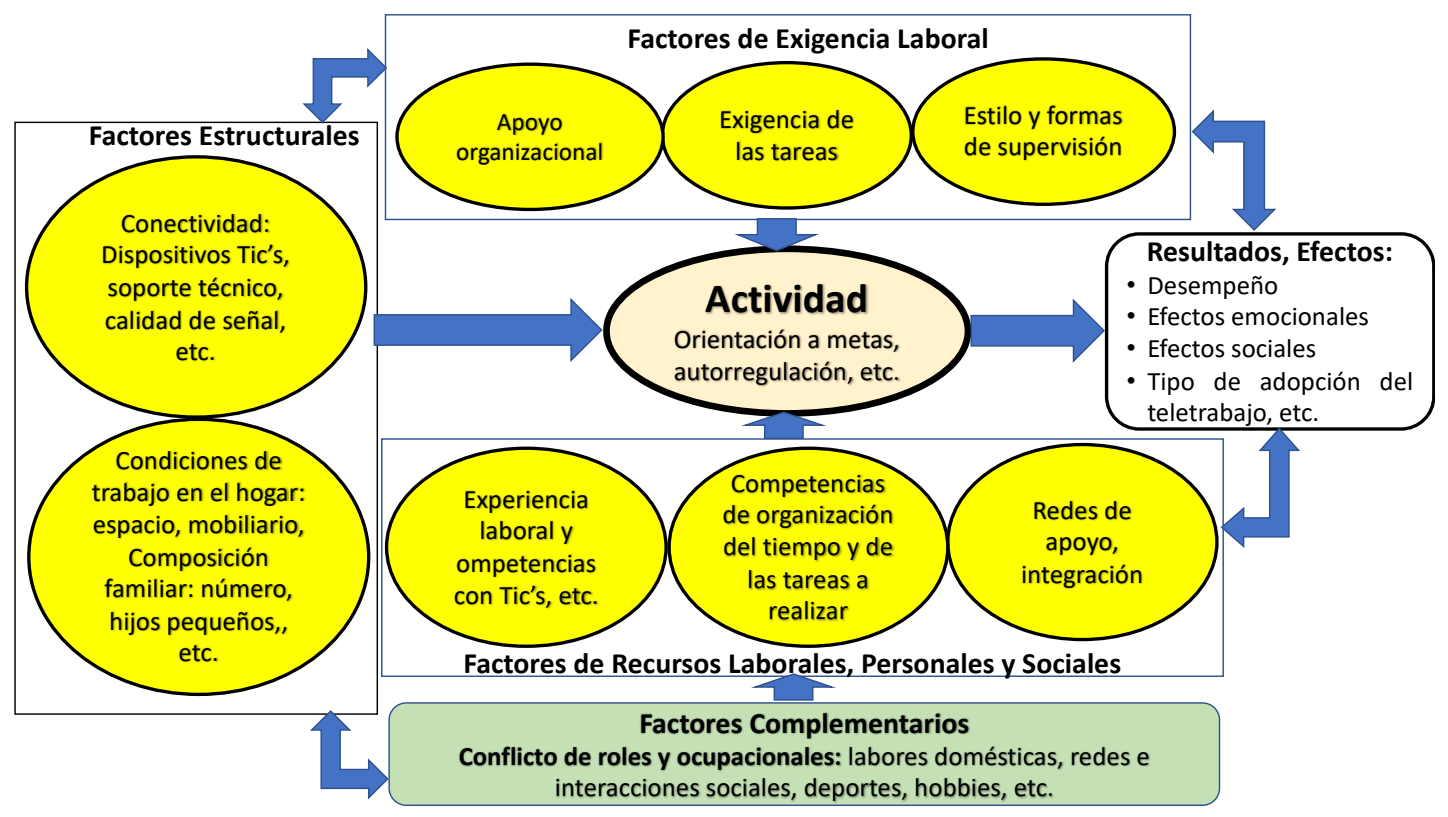

La sobreposición de las actividades laborales y del hogar configuran una doble tensión, pues si bien en ambas actividades se encuentran presentes dimensiones pragmáticas y relacionales, ellas juegan respectivamente roles fundacionales y de legitimación de distinto orden. En lo laboral, la dimensión pragmática es de naturaleza central como actividad orientada a fines, mientras que, en el hogar lo relacional y emocional constituyen la dimensión que da sentido sustantivo al quehacer compartido. Tales tensiones entre las prioridades que gobiernan estas distintas actividades, definirían parte relevante del contexto regulatorio de las demandas provenientes de ambas fuentes.

Fonner\& Stache (2012), apuntan que los teletrabajadores lidian con la tensión entre el deseo de flexibilidad y la necesidad de estructura, y utilizan señales y "ritos de paso" para ayudarse en la transición de roles. En contraste, algunos teletrabajadores preferirían estrategias integradoras, las que derivarían frecuentemente en confusión, interferencia y eventualmente conflicto entre estas dos esferas. El grado de identificación con un rol particular, diferencias individuales, la cultura, y fronteras institucionalizadas como, por 
ejemplo, horarios fijos de trabajo definidos por la organización, influirían en estas elecciones.

La segmentación entre roles permitiría sostener los límites entre roles, pero aumentaría la brecha del cambio de rol, requiriendo ritos que faciliten el tránsito. La integración entre roles, por su parte, disminuiría la brecha del cambio, pero dificultaría la formación y el mantenimiento de límites (Ashforth et al., 2000). Los teletrabajadores que realicen más transiciones del trabajo al hogar durante el horario laboral, tenderían a tener menores conflictos entre el trabajo y el hogar, pero un mayor conflicto entre el hogar y el trabajo. Los teletrabajadores que realicen más transiciones del hogar al trabajo, fuera del horario laboral, generarían más conflictos entre el trabajo y el hogar, y con las normas formales e implícitas de la organización (Palm et al., 2020; Kerman et al., 2021). Tales tendencias aparecían más marcadas en trabajo remoto que en situación de trabajo presencial (Delanoeije et al., 2019).

Se ha documentado igualmente que los teletrabajadores enfrentan presiones normativas a estar disponible para las exigencias y necesidades de la organización, forzando a acomodarse a horarios variables, trabajando más horas y más intensamente, y reportan aumentos de la carga de trabajo (Kelliher \& Anderson 2010), creando además un sentimiento de necesidad de estar siempre conectados, y eventualmente adicción al trabajo a través de la tecnología (Taskin \& Edwards, 2007). Estos fenómenos eventualmente gatillarían procesos de autorregulación necesarios para sostener la realización del trabajo (Taskin \& Devos, 2005).

La carga cognitiva podría constituirse a su vez en un gatillante del desarrollo de estrategias autorreguladoras, y sería en la calidad de estas estrategias, así como en los recursos que las personas disponen para llevarlas a cabo de manera efectiva y económica, que se jugaría la carga cognitiva resultante (Seufert, 2020). Así, la capacidad general de autorregulación de una persona incidiría en el éxito de su comportamiento, especialmente en el caso de cambios significativos en las condiciones de vida, la emergencia de nuevas tareas, y la necesidad de dominar tipos de actividad desconocidas o inusuales. El estudio realizado por Zinchenko et al. (2020) en el marco de la pandemia por COVID-19, muestra que, cuanto mayor sea el nivel de autorregulación consciente y de auto organización de su comportamiento desplegado por las personas, más productivas serían estas en situación de cuarentena. Cabe destacar que, el desarrollo de las capacidades autorregulatorias, no solo dependerían de la internalización de significados y estructuras procedurales ordenadoras del pensamiento y del comportamiento, sino que también de las estrategias de distribución y soporte de la actividad cognitiva y comportamental en medios externos (Cole \& Engeström, 1993).

\section{A modo de síntesis}

El teletrabajo ha estado sujeto a consideraciones en cuanto a sus potenciales efectos negativos en el plano del manejo del tiempo y de los espacios, producto de la superposición de la actividad laboral con los espacios de vida familiar y personal, conduciendo eventualmente a una extensión de las jornadas de trabajo, el trabajo nocturno, el aislamiento social, y mermas en las redes, intercambios y conocimientos necesarios para la actividad de las personas y la organización. Traduciéndose todo aquello en un incremento de la carga de trabajo resentida, del estrés y en una disminución de la productividad de las personas en el 
largo plazo.

La actual condición de implementación del teletrabajo, ha significado la incorporación forzada, masiva y heterogénea de roles laborales a esta modalidad, y mayoritariamente en condiciones de teletrabajo a tiempo completo, y se ha reportado que no sería universalmente beneficioso para todos los tipos de empleados, por lo que las organizaciones deberían considerar la posibilidad de diferenciar, por ejemplo, respecto al género y situación familiar (Feng \& Savani, 2020). La pandemia por COVID-19 ha permitido relevar la presencia de factores tales como, la condición de género, las demandas de cuidado de niños menores en casa, la distribución de roles domésticos, los requerimientos de herramientas y soporte tecnológico, las condiciones de espacio y equipamiento en el hogar, la naturaleza, contenidos y cargas del trabajo, las características y estilos de la supervisión, las instancias y modalidades de intercambio con colegas, el manejo de los tiempos de trabajo y del hogar, la planificación y la autorregulación, y el desarrollo de actividades sociales, la entretención y el ocio.

La relación entre trabajo remoto, el hogar y las organizaciones, constituye un desafío complejo, que implica hacerse cargo de la heterogeneidad existente. El equilibrio entre flexibilidad y estructura en el manejo de las demandas provenientes de variadas fuentes, es al parecer un aspecto crítico a resolver. El marco referencial y soporte que ofrezca la organización y las tecnologías, así como las reglas que se establezcan entre los teletrabajadores y sus supervisores, entre colegas, y entre los miembros del hogar, son a considerar para el despliegue virtuoso de procesos auto y co-regulatorios.

A su vez, la apropiación de los elementos estructurales del teletrabajo sería un factor determinante en los resultados de la actividad de los teletrabajadores (Errichiello et al., 2021). Esta apropiación podría estar vinculada a los procesos autorregulatorios y coregulatorios desplegados, sustentados en estrategias de modulación de las demandas en el tiempo, de uso de medios externos (TIC, alarmas y otros), de los espacios, la articulación de redes de intercambio y colaboración con colegas y supervisores, entre otros.

Tal escenario exige avanzar en estrategias que permitan adecuar las condiciones de realización del teletrabajo, logrando niveles apropiados de carga de trabajo, efectividad, y equilibrio entre las esferas laborales y personales.

Dicho esto, también es necesario visualizar las condiciones a considerar en un futuro escenario post pandemia, atendiendo a la probable extensión de las actuales condiciones de trabajo, en al menos un formato mixto o híbrido (Brunelle \& Fortin, 2021). Es muy posible que el retorno al trabajo bajo forma presencial sea gradual y bajo formatos variados, pues el regreso a un lugar de trabajo centralizado después de un largo período de teletrabajo, puede resultar estresante para algunas personas (Green et al., 2020), e implicará además particulares arreglos de infraestructura y organizacionales.

Según Green (2014), la adopción del teletrabajo posterior a la crisis provocada por los terremotos ocurridos en Christchurch, Nueva Zelandia, en 2010, permitió por medio de las TIC, la flexibilidad necesaria para equilibrar el trabajo con otras demandas. La productividad tendió a un incremento en las tareas autónomas, y un deterioro en las tareas colaborativas. Esto como efecto del aislamiento social en que se encontraban los teletrabajadores. En 
estudios posteriores Green et al. (2017 y 2020), constatan que, aunque en algunas organizaciones se extendió la práctica del teletrabajo una vez superada la crisis, su adopción tendió a ser baja, pues las organizaciones buscarían volver a sus comportamientos y rutinas anteriores. Este fenómeno de "normalización" de las prácticas laborales y organizacionales, quizás podría entenderse por la necesidad de las organizaciones de Christchurch, de acoplarse a las prácticas laborales generalizadas en el resto del mundo. No obstante, dada la condición de carácter mundial de la pandemia por COVID-19, y en la medida que esta se prolongue en el tiempo, la incorporación de las nuevas formas de trabajo generadas durante la pandemia, podrían tender a proyectarse más allá del término de la crisis, y eventualmente dar pie a la aparición de formas mixtas.

Hay que considerar igualmente que la abrupta e improvisada masificación del teletrabajo, como respuesta transitoria a la crisis, no ha sido homogénea en sus formas, según las organizaciones, naturaleza de las tareas, y en el tiempo. Tal situación, ha permitido tanto a las organizaciones, como a los trabajadores, experimentar y visualizar distintas posibilidades de realización de sus actividades, abriendo el abanico de alternativas con base a elementos reconocibles de sus respectivas bondades, limitaciones y factibilidades (Green et al., 2020).

\section{Conclusiones}

El despliegue futuro de las distintas alternativas, requerirá una especial atención, ya que no solo pondrán en tensión los equilibrios entre trabajo y vida personal, sino que supondrá igualmente velar por los efectos asociados en materia de procesos organizacionales, carga de trabajo, y de calidad de vida. Consistentemente, el desarrollo y adecuación de herramientas técnicas, organizacionales y de supervisión, necesarias para soportar apropiadamente la actividad de teletrabajo, así como las nuevas formas de trabajo presencial, estarán al centro de los desafíos del futuro cercano. Esto como condición para favorecer la consolidación de estrategias autorregulatorias y co-regulatorias adecuadas por parte de los trabajadores. En todos estos aspectos, la ergonomía tiene un rol relevante que cumplir, como disciplina orientada a adecuar el trabajo, sus herramientas y contextos, a las características de aquellos que lo realizan. 


\section{Referencias}

Annosi, M. C., Foss, N., Brunetta, F., \& Magnusson, M. (2017). The interaction of control systems and stakeholder networks in shaping the identities of self-managed teams. Organization Studies, 38(5), 619-645. https://doi.org/10.1177\%2F0170840616679454

Ashforth, B., Kreiner, G., \& Fugate, M. (2000). All in a day's work: Boundaries and micro role transitions. Academy of Management Review 25(3), 472-491. https://doi.org/10.5465/amr.2000.3363315

Baker, P. M. A., Moon, N. W., \& Ward, A. C. (2006). Virtual exclusion and telework: Barriers and opportunities of technocentric workplace accommodation policy. Work, 27(4), 421430. https://pubmed.ncbi.nlm.nih.gov/17148880/

Bedny, G. Z., Seglin, M. H., \& Meister, D. (2000). Activity theory: history, research and application. Theorical Issues In Ergonomics Science, 1(2), 168-206. https://doi.org/10.1080/14639220050171324

Bedny, G. Z., \& Karwowski, W. (2006). Activity theory as a basis for the study of work. Ergonomics, 47(2), 134-153.

Brunelle, E., \& Fortin, J. A. (2021). Distance makes the heart grow fonder: An examination of teleworkers' and office workers' job satisfaction through the lens of self-determination theory. Sage open. https://journals.sagepub.com/doi/10.1177/2158244020985516.

Chan, C. K. K. (2012). Co-regulation of learning in computer-supported collaborative learning $\begin{array}{lll}\text { environments: A discussion. Metacognition Learning, } & \text { A, 63-73. }\end{array}$ https://doi.org/10.1007/s11409-012-9086-Z

Chong, S. H., Huang, Y., \& Chang, Ch. H. (2020). Supporting interdependent telework employees: A moderated-mediation model linking daily covid-19 task setbacks to next-day work withdrawal. Journal of Applied Psychology. 105(12), 1408-1422. https://doi.org/10.1037/aploooo843

Cole, M., \& Engeström, Y. (1993). A cultural-historical approach to distributed cognition. En G. Salomon (ed.), Distributed cognitions: Psychological and educational considerations. Cambridge University Press.

Dahlstrom, T. R. (2013). Telecommuting and leadership style. Public Personnel Management, 42(3), 438-451. https://doi.org/10.1177/0091026013495731

De la Garza, C., \& Weill-Fassina, A. (2000). Régulations horizontales et verticales du risque. En A. Weill-Fassina \& T. H. Benchekroun (eds.), Le travail collectif: Perspectives actuelles en ergonomie (pp. 217-234). Octarès Éditions.

Delanoeije, J., Verbruggen, M., \& Germeys, L. (2019). Boundary role transitions: A day-to-day approach to explain the effects of home-based telework on work-to-home conflict and hometo-work conflict. Human Relations, 72(2),1843-1868. https://doi.org/10.1177\%2F0018726718823071

Díaz-Canepa, C. (2008). Organizaciones flexibles, incertidumbre e identidad. En A. Soto Roy (ed.), Flexibilidad Laboral y Subjetividades. LOM Ediciones.

Donnelly, N., \& Proctor-Thomson, S. B. (2015). Disrupted work: Home-based teleworking (HbTW) in the aftermath of a natural disaster. New Technology, Work and Employment. 3O(1), 47-61. https://doi.org/10.1111/ntwe.12040

Dumas, M., \& Ruiller, C. (2014). Le teletravail: les risques d'un outil de gestion des frontieres entre vie personnelle et vie professionnelle? Management \& Avenir, 74, 71-95. https://doi.org/10.3917/mav.074.0071 
Engeström, Y. (2008). The future of activity theory. En A. Sannino, H. Daniels \& K. Gutierrez, (eds), Learning and expanding with activity theory. Cambridge University Press.

Errichiello, L., \& Pianese, T. (2021). The role of organizational support in effective remote work implementation in the Post-COVID era. En Handbook of Research on Remote Work and Worker Well-Being in the Post-COVID-19 Era (pp. 221-242) https://doi.org/10.4018/978-17998-6754-8.cho13

Felstead, A., Jewson, N., \&Walters, S. (2003). Managerial control of employees working at home. British Journal of Industrial Relations, 41(2), 241-264. https://doi.org/10.1111/14678543.00271

Feng, Z., \& Savani, K. (2020). Covid-19 created a gender gap in perceived work productivity and job satisfaction: Implications for dual-career parents working from home. Gender in Management, 35(7/8), 719-736. https://doi.org/10.1108/GM-07-2020-0202

Fischer, T., Küll, S., Niederländer, U., \& Stabauer, M. (2021). The new normal? Motivators for and hindrances to telework. International Conference on Human-Computer Interaction, HCII 2021: HCI in Business, Government and Organizations (pp. 327-346).

Fonner, K. L., \& Stache, L. C. (2012). All in a day's work, at home: Teleworkers' management of micro role transitions and the work-home boundary. New Technology, Work and Employment, 27(3), 242-257. https://doi.org/10.1111/j.1468-005X.2012.00290.x

Gajendran, R. S., \& Harrison, D. A., \& Delaney-Klinger, K. (2015). Are telecommuters remotely good citizens? Unpacking telecommuting's effects on performance via I - deals and job resources. Personnel Psychology, 68(2), 353-393. https://doi.org/10.1111/peps.12082

Golden, T. D. (2007). Coworkers who telework and the impact on those in the office: Understanding the implications of virtual work for coworker satisfaction and turnover intentions. Human $\quad$ Relations, $60(11), \quad 1641-1667$. https://doi.org/10.1177/0018726707084303

Golden, T. D., \& Raghuram, S. (2010). Teleworker knowledge sharing and the role of altered relational and technological interactions. Journal of Organizational Behavior, 31(8), 10611085. https://doi.org/10.1002/job.652

Green, N. J. (2014). A multi-level analysis of telework adoptions and outcomes following a natural disaster: The experience of two Christchurch organisations [tesis de maestría, Auckland University of Technology]. Repositorio AUT. http://hdl.handle.net/10292/7981

Green, N., Tappin, D., \& Bentley, T. (2017). Exploring the teleworking experiences of organisations in a post-disaster environment. New Zealand Journal of Human Resources Management, 17(1), 1-19.

Green, N., Tappin, D., \& Bentley, T. (2020). Working from home before, during and after the Covid-19 pandemic: implications for workers and organizations. New Zealand Journal of Employment Relations, 45(2), 5-16. https://doi.org/10.24135/nzjer.v45i2.19

Hadwin, A. F., Järvelä, S., \& Miller, M. (2017). Self-regulation, co-regulation and shared regulation in collaborative learning environments. En D. Schunk \& J. Greene, (eds.), Handbook of Self-Regulation of Learning and Performance. Routledge.

Henry, M.S., Le Roux, D. B., \& Parry, D. A. (2021). Working in a post Covid-19 world: Towards a conceptual framework for distributed work. South African Journal of Business Management, 52(1), a2155. https://doi.org/10.4102/sajbm.v52i1.2155

Hughes, K. D. \& Silver, W. A. (2020). Beyond time-binds: Rethinking work-family dynamics for a mobile world. Human Relations, 73(7), 924-952.

Järvelä, S., Kirschner, P.A., Hadwin, A., Järvenoja, H., Malmberg, J., Miller, M., \& Laru, J., (2016). Socially shared regulation of learning in CSCL: Understanding and prompting 
individual- and group-level shared regulatory activities. International Journal of ComputerSupported Collaborative Learning, 11(3), 263-280. https://www.learntechlib.org/p/175393/

Kelliher, C., \& Anderson, D. (2010). Doing more with less? Flexible working practices and the intensification of work. Human Relations, 63(1), 83-106. https://doi.org/10.1177/0018726709349199

Kerman, K., Korunka, Ch., \& Tement, S. (2021). Work and home boundary violations during the COVID-19 pandemic: The role of segmentation preferences and unfinished tasks. Applied Psychology, 1-23. https://doi.org/10.1111/apps.12335

Madlock, P. E. (2012). The influence of supervisors' leadership style on telecommuters. Journal of Business Strategies, 29(1), 1-24. https://doi.org/10.54155/jbs.29.1.1-24

Mazmanian, M., Orlikowski, W. J., \& Yates, J. (2013). The autonomy paradox: The implications of mobile email devices for knowledge professionals. Organization Science, 24(5), 1337-1357. https://doi.org/10.1287/orsc.1120.0806

Morosanova, V. I., Gaidamashko, I. V., Chistyakovac, S. N., Nailia, S. V., Kondratyuk, N. G. \& Burmistrova-Savenkova, A.V. (2017). Regulatory and personality predictors of the reliability of professional actions. Psychology in Russia: State of the Art, 10(4), 195-208. http://psychologyinrussia.com/volumes/?article $=6945$

Nansen, B., Arnold, M., Gibbs, M., \& Davis, H. (2010). Time, space, and technology in the working-home: An unsettled nexus. New Technology, Work, and Employment, 25(2), 136153. http://hdl.handle.net/11343/29985

Nückles, M., Roelle, J., Glogger-Frey, I., Waldeyer, J., \& Renkl, A. (2020). The self-regulationview in writing-to-learn: Using journal writing to optimize cognitive load in self-regulated learning. Educational Psychology Review, 32, 1089-1126. https://doi.org/10.1007/s10648020-09541-1

Olivares, G., Villalobos, V., Rodríguez, C., Cerda, L., Besoaín, S., Díaz, C., Méndez, I., Indo, I., Pinto, R., Herrera, S., Cerda, E., \& Román, L. (2020). Guía de implementación de teletrabajo. Laboratorio de Ergonomía, Departamento de Kinesiología, Facultad de Medicina, Universidad de Chile. https://libros.uchile.cl/1093

Palm, E., Seuber, Ch., \& Glaser, J. (2020). Understanding employee motivation for work-tononwork integration behavior: A reasoned action approach. Journal of Business and Psychology, 35, 683-696. https://doi.org/10.1007/s10869-019-09648-5

Peters, P., \& van der Lippe, T. (2007). The time-pressure reducing potential of telehomeworking: The Dutch case. The International Journal of Human Resource Management, 18(3), 430447. https://doi.org/10.1080/09585190601167730

Peters, P., Wetzels, C., \& Tijdens, K. (2008). Telework: Timesaving or time-consuming? An investigation into actual working hours. Journal of Interdisciplinary Economics, 19(4), 421442. https://doi.org/10.1177/02601079X08001900407

Rabardel, P. (1995). Les hommes et les technologies: Approche cognitive des instruments contemporains. Armand Colin.

Sanda, M. A. (2018). Mediating subjective task complexity in job design: A critical reflection of historicity in self-regulatory activity. En C. Baldwin (ed.), Advances in Neuroergonomics and Cognitive Engineering. Springer International Publishing. https://doi.org/10.1007/978-3319-60642-2 32

Seufert, T. (2020). Building bridges between self-regulation and cognitive load - An invitation for a broad and differentiated attempt. Educational Psychology Review, 32, 1151-1162. https://doi.org/10.1007/s10648-020-09574-6 
Sewell, G., \& Taskin, L. (2015). Out of sight, out of mind in a new world of work? Autonomy, control, and spatiotemporal scaling in telework. Organization Studies, 36(11), 1507-1529. https://doi.org/10.1177/0170840615593587

Taskin, L. (2010). La déspatialisation. Enjeu de gestion. Revue française de gestion, 36(202), 6176. https://doi.org/10.3166/RFG.202.61-76

Taskin, L., \& Devos, V. (2005). Paradoxes from the individualization of human resource management: The case of telework. Journal of Business Ethics, 62(1), 13-24. https://doi.org/10.1007/s10551-005-8710-0

Taskin, L., \& Edwards, P. (2007). The possibilities and limits of telework in a bureaucratic environment: Lessons from the public sector. New Technology, Work and Employment, 22(3), 195-207. https://doi.org/10.1111/j.1468-005X.2007.00194.x

Thulin, E., Vilhelmson, B., \& Johansson, M. (2019). New telework, time pressure, and time use control in everyday life. Sustainability, 11(11), 3067. https://doi.org/10.3390/su11113067

Van der Lippe, T., \& Lippényi, Z. (2019). Co-workers working from home and individual and team performance. New Technology, Work and Employment, 35(1), 60-79. https://doi.org/10.1111/ntwe.12153

Volet, S., Summers, M., \& Thurman, J. (2009). High-level co-regulation in collaborative learning: How does it emerge and how is it sustained? Learning and Instruction, 19(2), 128-143. https://doi.org/10.1016/j.learninstruc.2008.03.001

Vygotsky, L. S. (1981). The instrumental method in psychology. En J. V. Wertsch, (ed.), The concept of activity in Soviet psychology (pp. 134-143). M. E. Sharpe Publishers.

Vygotsky, L. S. (1997). Pensée et langage. La Dispute.

Wang, B., Liu, Y., Qian, J., \& Parker, S. K. (2020). Achieving effective remote working during the COVID-19 pandemic: A work design perspective. Applied Psychology, 70(1), 16-59. https://doi.org/10.1111\%2Fapps.12290

Winne, P. H. (2019). Paradigmatic dimensions of instrumentation and analytic methods in research on self-regulated learning. Computers in Human Behavior, 96, 285-289. https://doi.org/10.1016/j.chb.2019.03.026

Winters, F. I., Greene, J. A., \& Costich, C. M. (2008). Self-regulation of learning within computerbased learning environments: A critical analysis. Educational Psychology Revue, 20, 429444. https://doi.org/10.1007/s10648-008-9080-9

Wirth, J., Stebner, F., Trypke, M., Schuster, C., \& Leutner, D. (2020). An interactive layers model of self-regulated learning and cognitive load. Educational Psychology Review, 32, 1127-1149. https://doi.org/10.1007/s10648-020-09568-4

Zheng, X. L., Kim, H. S., Lai, W. H., \& Hwang, G. J. (2020). Cognitive regulations in ICTsupported flipped classroom interactions: An activity theory perspective. British Journal of Educational Technology, 51(1), 103-130. https://doi.org/10.1111/bjet.12763

Zinchenko, Y. P., Morosanova, V. I., Kondratyuk, N. G., \& Fomina, T. G. (2020). Conscious selfregulation and self-organization of life during the COVID-19 pandemic. Psychology in Russia: State of the Art, 13(4), 168-182. http://psychologyinrussia.com/volumes/?article $=8867$ 